Draft Version November 18, 2016

Preprint typeset using $\mathrm{L}_{\mathrm{T}} \mathrm{EX}$ style emulateapj v. 01/23/15

\title{
DISCOVERY OF XO-6b: A HOT JUPITER TRANSITING A FAST ROTATING F5 STAR ON AN OBLIQUE
} ORBIT

\author{
N Crouzet ${ }^{1}$, P. R. McCullough ${ }^{2}$, D. Long ${ }^{3}$, P. Montanes Rodriguez ${ }^{4}$, A. Lecavelier des Etangs ${ }^{5}$, I. Ribas $^{6}$, \\ V. Bourrier ${ }^{7}$, G. Hébrard ${ }^{5}$, F. Vilardell ${ }^{8}$, M. Deleuil ${ }^{9}$, E. Herrero ${ }^{6,8}$, E. Garcia-Melendo ${ }^{10,11}$, \\ L. Akhenak ${ }^{5}$, J. Foote ${ }^{12}$, B. Gary ${ }^{13}$, P. Benni ${ }^{14}$, T. Gulllot ${ }^{15}$, M. Conjat ${ }^{15}$, D. Mékarnia ${ }^{15}$, J. Garlitz ${ }^{16}$, \\ C. J. Burke ${ }^{17}$, B. Courcol ${ }^{9}$, and O. Demangeon ${ }^{9}$ \\ ${ }^{1}$ Dunlap Institute for Astronomy \& Astrophysics, University of Toronto, 50 St. George Street, Toronto, Ontario, Canada M5S 3H4 \\ 2 Department of Physics and Astronomy, Johns Hopkins University, 3400 North Charles Street, Baltimore, MD 21218, USA \\ 3 Space Telescope Science Institute, 3700 San Martin Dr, Baltimore, MD 21218, USA \\ ${ }^{4}$ Instituto de Astrofísica de Canarias, C/Vía Láctea s/n, E-38200 La Laguna, Spain \\ ${ }^{5}$ Institut d'Astrophysique de Paris, UMR7095 CNRS, Université Pierre \& Marie Curie, 98bis boulevard Arago, 75014 Paris, France \\ ${ }^{6}$ Institut de Ciències de l'Espai (CSIC-IEEC), Campus UAB, Carrer de Can Magrans s/n, 08193 Bellaterra, Spain \\ ${ }^{7}$ Observatoire de l'Université de Genève, 51 chemin des Maillettes, 1290 Sauverny, Switzerland \\ 8 Observatori del Montsec (OAdM), Institut d'Estudis Espacials de Catalunya (IEEC), Gran Capità, 2-4, Edif. Nexus, 08034 Barcelona, \\ ${ }_{9}^{9}$ Aix Marseille Université, CNRS, LAM (Laboratoire d'Astrophysique de Marseille) UMR 7326, 13388 Marseille, France \\ 10 Departamento de Física Aplicada I, Escuela Técnica Superior de Ingeniería, Universidad del País Vasco UPV/EHU, Alameda Urquijo \\ s/n, 48013 Bilbao, Spain \\ 11 Fundacio Observatori Esteve Duran, Avda. Montseny 46, Seva E-08553, Spain \\ 12 Vermillion Cliffs Observatory, 4175 E. Red Cliffs Drive, Kanab, UT 84741, USA \\ 13 Hereford Arizona Observatory, 5320 East Calle de la Manzana, Hereford, AZ 85615, USA \\ 14 Acton Sky Portal, Acton, MA, USA \\ 15 Laboratoire Lagrange, Université Côte d'Azur, Observatoire de la Côte d'Azur, CNRS, Boulevard de l'Observatoire, CS 34229, \\ F-06304 Nice Cedex 4, France \\ 16 Elgin Observatory, Elgin, OR, USA and \\ 17 SETI Institute/NASA Ames Research Center, Moffett Field, CA 94035, USA \\ Draft version November 18, 2016
}

\begin{abstract}
Only a few hot Jupiters are known to orbit around fast rotating stars. These exoplanets are harder to detect and characterize and may be less common than around slow rotators. Here, we report the discovery of the transiting hot Jupiter XO-6b, which orbits a bright, hot, and fast rotating star: $\mathrm{V}=$ $10.25, T_{e f f \star}=6720 \pm 100 \mathrm{~K}, v \sin i_{\star}=48 \pm 3 \mathrm{~km} \mathrm{~s}^{-1}$. We detected the planet from its transits using the XO instruments and conducted a follow-up campaign. Because of the fast stellar rotation, radial velocities taken along the orbit do not yield the planet's mass with a high confidence level, but we secure a 3- $\sigma$ upper limit $M_{p}<4.4 \mathrm{M}_{\mathrm{Jup}}$. We also obtain high resolution spectroscopic observations of the transit with the SOPHIE spectrograph at the 193-cm telescope of the Observatoire de Haute-Provence and analyze the stellar lines profile by Doppler tomography. The transit is clearly detected in the spectra. The radii measured independently from the tomographic analysis and from the photometric lightcurves are consistent, showing that the object detected by both methods is the same and indeed transits in front of XO-6. We find that XO-6b lies on a prograde and misaligned orbit with a sky-

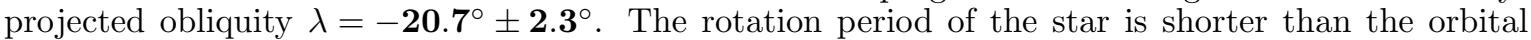
period of the planet: $P_{\text {rot }}<2.12$ days, $P_{\text {orb }}=3.77$ days. Thus, this system stands in a largely unexplored regime of dynamical interactions between close-in giant planets and their host stars.

Keywords: Stars: planetary systems — Planets and satellites: individual (XO-6b) — Methods: observational — Techniques: photometric — Techniques: spectroscopic
\end{abstract}

\section{INTRODUCTION}

Most of our understanding of close-in gas giant planets is enabled by studying those transiting in front of bright stars. Such systems are favorable to photometric and radial velocity observations and to atmospheric characterization. The vast majority of these systems have been discovered by ground-based surveys such as WASP (Pollacco et al. 2006, Collier Cameron et al. 2007) and HAT (Bakos et al. 2004). These two projects detected a total of $\sim 60$ hot Jupiters around stars of magnitude $J<10$. The CoRoT and Kepler missions provided little addition to this sample because they target fainter stars ; $K e$ pler discovered two planets around stars of magnitude

crouzet@dunlap.utoronto.ca
$J<10$ with a radius larger than $0.3 \mathrm{R}_{\mathrm{Jup}}$ : Kepler$16 \mathrm{~b}$ and Kepler-25c. The ratio of such hot Jupiters numbers (60:2) is approximately the same as the corresponding ratio of survey areas on the sky (30,000:100 square degrees).

Among parameters used to characterize hot Jupiters, the distribution of obliquities draws particular attention. Sky-projected obliquities can be measured by observing exoplanets in spectroscopy during a transit: the planet occults a portion of its rotating host star and distorts the apparent stellar line profile ; this effect is known as the Rossiter-McLaughlin effect (Holt 1893: Rossiter 1924 McLaughlin 1924). It is most commonly analyzed through velocimetry. A more sophisticated technique, Doppler tomography, has also been used in a few 
cases and provides more precise parameters as well as a wider range of information on the planet and the host star (e.g. Collier Cameron et al. 2010a b; Bourrier et al. 2015). With the rapid growth of Rossiter-McLaughlin measurements in recent years, obliquities have become a powerful way to probe different theories for the dynamical history of hot Jupiters. Winn et al. (2010) noted that hot Jupiters orbiting stars with relatively cool photospheres $(\mathbf{T}<\mathbf{6 2 5 0} \mathrm{K})$ have low obliquities whereas those orbiting hotter stars show a wide range of obliquities. This trend has been investigated in the context of dynamical interactions (e.g. Schlaufman 2010, Albrecht et al. 2012; Dawson \& Chiang 2014; Winn \& Fabrycky 2015). The comparison of planets' orbital periods and host stars' rotation periods also constrains the dynamics of hot Jupiters (Mazeh et al. 2005; Pont 2009; Husnoo et al. 2012). However, the vast majority of known hot Jupiters orbit around slowly rotating stars. This trend is supported by a dearth of Kepler Objects of Interest at short orbital periods around fast rotating stars (McQuillan et al. 2013) ; such studies could be used to calibrate potential selection effects of ground-based transit surveys against detecting planets around fast rotating stars.

The XO project (McCullough et al. 2005) aims at detecting transiting exoplanets around bright stars from the ground with small telescopes. The project started in 2005 and discovered five close-in gas giant planets, $\mathrm{XO}-1 \mathrm{~b}$ to $\mathrm{XO}-5 \mathrm{~b}^{1}$. A new version of $\mathrm{XO}$ was deployed in 2011 and 2012 and operated nominally from 2012 to 2014. In this paper, we report the discovery of XO-6b, a transiting hot Jupiter orbiting a bright and fast rotating star. After its detection by the XO instruments, we conducted a follow-up campaign via photometry, radial velocity, and Rossiter-McLaughlin observations including a Doppler tomography analysis in order to establish the presence of the planet and extract the system parameters.

The XO setup and observations are described in Section 2 and the data reduction pipeline in Section 3 . The follow-up campaign and the nature of the XO-6 system are presented in Section 4 . The stellar parameters are derived in Section 5. In Section 6, we discuss the properties of the XO-6 system in the context of dynamical studies of hot Jupiters and their host stars, and we conclude in Section 7.

\section{DISCOVERY INSTRUMENTATION AND OBSERVATIONS}

The new XO instrumental setup consists of three identical units installed at Vermillion Cliffs Observatory, Kanab, Utah, at Observatorio del Teide, Tenerife, Canary Islands, and at Observatori Astronòmic del Montsec (OAdM), near Àger, Spain. Each unit is composed of two $10 \mathrm{~cm}$ diameter and $200 \mathrm{~mm}$ focal length Canon telephoto lenses equipped with an Apogee E6 $1024 \times 1024$ pixels CCD camera mounted on a German-Equatorial Paramount ME mount and protected by a shelter with a computer-controlled roof. All six lenses and cameras operate in a network configuration and point towards the same fields of view. These fields of view are different from those of the original XO, with no overlap. Instrumental parameters and data reduction methods are

1 McCullough et al. (2006); Burke et al. (2007); Johns-Krull et al. (2008); McCullough et al. (2008); Burke et al. (2008) similar as in McCullough et al. (2005) unless indicated. The focus is adjusted to yield a PSF (Point Spread Function) FWHM (Full Width Half Maximum) of $\sim 1$ pixel; in practice the FWHM varies between 1 and 1.5 pixels. The CCDs are used in spatial-scan mode: pixels are read continuously while stars move along on the detector. The mount tracking and CCD reading rates are calculated to yield round PSFs. The resulting images are long strips of $43.2^{\circ} \times 7.2^{\circ}$ instead of $7.2^{\circ} \times 7.2^{\circ}$ images that would result if the CCD was used in staring mode. This technique reduces overheads, increases the time spent at collecting photons, and maximizes the number of observed bright stars. The same two strips of the sky were observed over and over. Between 50 and 100 strips were recorded each night by each camera; the exposure time is $317 \mathrm{~s}$ for a full strip ( $53 \mathrm{~s}$ for each $7.2^{\circ} \times 7.2^{\circ}$ region). A weather sensor records meteorological data such as the ambient temperature, sky temperature, humidity, rain drops, wind speed and direction, dew point, etc... every minute. Each unit operates robotically: the weather data are interpreted in real time, a command is sent to open or close the roof, and run the observations. The units can also be controlled remotely. A rotating webcam sensitive in the visible and near-infrared is mounted inside each shelter and gives a live view of the systems. The observations span a duration of two times nine months between 2012 and 2014, with gaps due only to weather and instrumental problems.

\section{DATA REDUCTION}

First, data taken by the six camera systems are reduced independently. The strips are carved into $1024 \times 1024$ pixel images, and we discard the first $1024 \times 1024$ image of each strip where the scanning is just starting. This yields 9 fields of $7.2^{\circ} \times 7.2^{\circ}$; the field located around the celestial North pole is covered by both strips and has the maximum phase coverage. An approximate WCS (World Coordinate System) solution is found for each image using the astrometry.net software program ${ }^{2}$, and it is improved using a 6 parameter astrometric solution.

Dark frames are taken at the beginning and end of each night and are averaged to yield one dark per night. A flat-field is built from twilight flats and is used for the whole duration of the observations. Science frames are calibrated by the dark and flat. Warm columns are identified using high-pass filters; columns with a excess of 15 ADU with respect to their 10 neighbouring columns are flagged and their excess flux is removed. We find typically between 0 and 5 warm columns per image, except for one CCD which has up to 100 warm columns.

We select the target stars from a reference image taken under very good conditions. This yields about 6000 stars in each field. To identify the target stars on each image, the coordinates of point sources are correlated to that of the target stars in the reference image. We perform circular aperture photometry using the Stellar Photometry Software program (Janes \& Heasley 1993). The sky background is calculated in an annulus around each star and is subtracted. The optimum photometric aperture radius is computed as a function of stellar magnitude for each camera using data taken under average conditions, and is kept constant for each star; this radius varies from

\footnotetext{
2 http://astrometry.net/
} 
2 to 10 pixels depending on the star magnitude. The photometric measurements are gathered in a star-epoch array where stars are sorted by ascending instrumental magnitude. One array is obtained for each field and each camera.

The next reduction steps are performed only on the 2000 brightest stars of each field, which corresponds approximately to a limit magnitude $\mathrm{V}=12$ and a $1 \% \mathrm{RMS}$ photometric precision. Several calibrations are applied independently for each scan direction (the strips can be observed scanning North or South). The mean magnitude of each star is subtracted; we use the magnitude residuals in all the following. We select the reference stars for each star and each scan direction separately. For a given star $s$, we subtract the photometric time series of $s$ from those of all the stars, and evaluate the mean absolute deviation (MAD) of the resulting time series. Stars are sorted by increasing MAD; the first index is excluded because it corresponds to star $s$, and the following $\mathbf{N}$ stars are kept as reference stars. Then, we build a reference time-series using an outlier resistant mean of the $\mathrm{N}$ reference stars, and subtract it to the time-series of star $s$. This yields a calibrated lightcurve for $\operatorname{star} s$. We use $\mathbf{N}=\mathbf{1 0}$ for all the stars. Then, for each epoch, we remove a 3rd oder polynomial corresponding to low-frequency variations of magnitude residuals in the CCD's $x, y$ plane, also called "L-flats". We also remove a linear dependance of each lightcurve with airmass.

Bad data are flagged according to several criteria: the quality of each epoch measured from the dispersion of the residuals of a subset of stars, the sky brightness and its homogeneity (which account for clouds or a shelter's wall partially obscuring the field of view), the airmass, the number of detected stars, and the image cross-correlation (revealing for example double point sources due to an imperfect tracking occurring occasionally on one of the mounts). Then, systematic effects are removed using the Sysrem algorithm (Tamuz et al. 2005). Finally, the lightcurves from the 6 cameras are combined together and we search for periodic signals using the BLS (Box Least Square) algorithm (Kovács et al. 2002). We keep signals compatible with planetary transits for visual inspection and detailed investigation.

The discovery lightcurve of XO-6b is shown in Figure 1. These data were obtained from the three units (six cameras) of the new XO instruments during the first campaign, from fall 2012 to spring 2013.

\section{FOLLOW-UP CAMPAIGN}

We conducted follow-up observations and analyses in photometry and spectroscopy to establish the nature of the system. The first spectroscopic measurements reveal that the star is a fast rotator, which jeopardizes measuring the mass of the companion by radial velocities (see Section 4.3). Therefore, particular care is given in the photometric follow-up analysis to look for signs of a stellar eclipsing binary or a triple star system, as detailed in the following. Although the XO lightcurve is contaminated by another star which is 1 magnitude fainter and located at 38" separation from XO-6, both stars are well resolved by the follow-up instruments. Nine other stars are located within 1 arcminute of XO-6 and

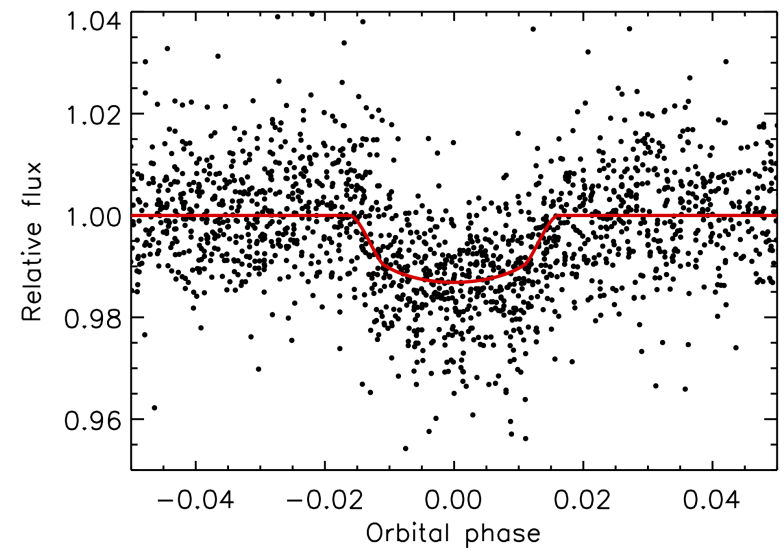

Figure 1. Discovery lightcurve of XO-6b (black dots) and best transit fit using the parameters from Table 5 (red line).

are 4.5 to 7 magnitude fainter; they do not affect the follow-up measurements.

\subsection{Photometric follow-up}

The photometric follow-up observations were conducted by an extended team of amateur and professional astronomers with facilities summarized in Table 1. We observed 18 transit events with good quality data, from December 26th, 2013 to February 2nd, 2015 using different filters (g', r', i', B, V, R, I); some transits were observed alternating between two filters. In total, this yields 26 sets of individual transits and filters that we use in the analysis (Figure 2).

\subsubsection{Analysis of individual transits}

First, each transit time series is analyzed individually in order to search for signs of false-positive configurations (such as differences between even and odd transits or large transit timing variations) and to refine the ephemeris. Julian dates are converted to BJD (Barycentric Julian Dates). Each lightcurve is fitted by a combination of a transit model from Mandel \& Agol (2002) and a linear trend to correct for systematic effects. We assume a circular orbit. We use the downhill simplex minimization procedure with the following parameters: $P, T_{0}$, $a / R_{\star}, R_{p} / R_{\star}, i$, and $t_{0}$, where $P$ is the orbital period, $T_{0}$ the average mid-transit time, $a$ the semi-major axis, $R_{\star}$ the stellar radius, $R_{p}$ the planetary radius, $i$ the inclination, and $t_{0}$ the offset of the mid-transit time of each transit with respect to $T_{0}$. We include two more parameters to account for a flux offset and a linear trend. We calculate the limb-darkening coefficients using the John Southworth's JKTLD software program (Southworth 2015) ${ }^{3}$ with the Claret (2000, 2004) tables, the ATLAS model for the stellar atmosphere, a quadratic limb-darkening law, and the stellar parameters $T_{e f f \star}=6720 \mathrm{~K}, \log g_{\star}$ $=4.036, M / H=0$, and $V_{\text {micro }}=2 \mathrm{~km} \mathrm{~s}^{-1}$ (see Section 5). We fix these coefficients to their theoretical values in each bandpass. We remove outliers lying away from the transit model by more than $3 \sigma$, where $\sigma$ is the standard deviation of the lightcurve. The individual lightcurves are displayed in Figure 2

We improve the transit ephemeris parameters $P$ and $T_{0}$ in an iterative process. First, $t_{0}$ is fixed to 0 whereas

\footnotetext{
3 http://www.astro.keele.ac.uk/jkt/codes/jktld.html
} 
Table 1

Observatories and telescopes used for the photometric follow-up.

The diameters of the primary mirrors are given in inches and $\mathrm{cm}$.

\begin{tabular}{lll}
\hline \hline Observatory & Telescope & Label \\
\hline Hereford Arizona Observatory, Arizona, USA & Celestron 11" (28 cm) - Meade 14" (36 cm) & HAO \\
Acton Sky Portal, Massachusetts, USA & $11 "(28 \mathrm{~cm})$ & ASP \\
Observatori Astronòmic del Montsec, Catalonia, Spain & Joan Oró Telescope 31" (80 cm) & TJO \\
Observatoire de Nice, France & Schaumasse 16" (40 cm) & NCE \\
Vermillion Cliffs Observatory, Kanab, Utah, USA & $24 "(60 \mathrm{~cm})$ & VCO \\
Elgin Observatory, Elgin, Oregon, USA & $12 "(30 \mathrm{~cm})$ & EO \\
\hline \hline
\end{tabular}

$a / R_{\star}, R_{p} / R_{\star}$, and $i$ are free for each transit. Then, $a / R_{\star}$, $R_{p} / R_{\star}$, and $i$ are fixed to their mean over all transits and $t_{0}$ is allowed to vary. Finally, we calculate a linear fit between the set of individual $t_{0}$ and the period index, and we update $P$ and $T_{0}$ in order to remove this linear variation. This new ephemeris as well as the new values of $a / R_{\star}, R_{p} / R_{\star}$, and $i$ are used as an initial guess for the next iteration. We perform 100 iterations. The final distributions for the parameters are well behaved, and we use the median values of the distributions of $P$ and $T_{0}$ as the final ephemeris. We compute the uncertainties in the ephemeris in two ways. A first estimate is obtained from the standard deviation of these $P$ and $T_{0}$ distributions. A second estimate is obtained using the residual-permutation method (see Pont et al. 2006, and references therein), in which we shift the $t_{0}$ residuals with respect to the period index, calculate a new linear fit, and derive a new ephemeris. Applying all possible shifts results in distributions for $P$ and $T_{0}$, and their standard deviations yield the uncertainties on these parameters, which are larger than the first estimate by a factor of 1.2 and 1.5 for $P$ and $T_{0}$ respectively. We keep the larger values, obtained from the residualpermutation method, as the final uncertainties. The final values and uncertainties of $P$ and $T_{0}$ are reported in Table 5. This ephemeris is in excellent agreement with the original XO lightcurve. In this analysis, we find no evidence for transit timing variations and no significant difference between odd and even transits at the level of precision of our data.

\subsubsection{Analysis of transits per bandpass}

We gather data and fit a transit model in each bandpass $(\mathrm{B}, \mathrm{V}, \mathrm{R}, \mathrm{I})$. We fix $P$ and $T_{0}$ to the values derived in Section 4.1.1, and the limb-darkening coefficients to their theoretical values in each bandpass. The free parameters are $a / R_{\star}, R_{p} / R_{\star}$, and $i$, plus a flux offset and a linear trend. We assume a circular orbit and use the downhill simplex minimization procedure. Then, the uncertainties are calculated using a customized residualpermutation method: instead of shifting the residuals over the whole transit curve, we divide this curve into two parts from mid-transit, and for each shift on one side all shifts are successively applied on the other side. This results in $\mathcal{O}\left(N^{2}\right)$ permutations instead of $\mathcal{O}(N)$, where $N$ is the number of data points, and allows correlated noise features to be shuffled with respect to each other before and after the transit. The best transit model is calculated for each permutation using a Levenberg-Marquardt minimization method, and the standard deviations of the posterior distributions yield the 1- $\sigma$ uncertainties on the transit parameters. These parameters are consistent be- tween the different bandpasses within their uncertainties (Table 2 Figure 3). We obtain the final transit parameters by a weighted average of all bandpasses with weights of $1 / \sigma^{2}$ where $\sigma$ is the uncertainty in each bandpass.

Table 2

Transit parameters of the XO-6 system obtained in each photometric bandpass and their weighted average.

\begin{tabular}{cccc}
\hline \hline Bandpass & $a / R_{\star}$ & $R_{p} / R_{\star}$ & $i[\mathrm{deg}]$ \\
\hline B & $9.21 \pm 0.48$ & $0.1179 \pm 0.0026$ & $85.94 \pm 0.51$ \\
V & $9.01 \pm 0.35$ & $0.1151 \pm 0.0017$ & $86.02 \pm 0.39$ \\
R & $9.07 \pm 0.41$ & $0.1153 \pm 0.0019$ & $85.90 \pm 0.42$ \\
I & $9.49 \pm 0.36$ & $0.1114 \pm 0.0017$ & $85.94 \pm 0.33$ \\
Average & $9.20 \pm 0.19$ & $0.1144 \pm 0.0013$ & $85.95 \pm 0.20$ \\
\hline \hline
\end{tabular}

\subsection{Secondary eclipse}

We do not detect the secondary eclipse in the XO lightcurve, but we can constrain the eclipse depth $\delta_{e}$ in the XO bandpass assuming a circular orbit. First, we calculate an upper limit on $\delta_{e}$ as $\sigma / \sqrt{N}$, where $\sigma$ and $N$ are the standard deviation and number of in-eclipse points respectively: we find $\delta_{e}<0.114 \%$ at $3-\sigma$. However, this calculation assumes that only white noise is affecting the XO lightcurve. In a second approach, we estimate the noise from the data themselves. We eliminate the in-transit points, fold the lightcurve at $10^{4}$ different periods ranging from 3 to 4.5 days, split each lightcurve into segments corresponding to the eclipse duration, and calculate the mean flux in each segment. This yields a distribution that represents the flux variations over durations equivalent to that of the eclipse. The $3-\sigma$ values of this distribution yield the $3-\sigma$ upper limit on $\delta_{e}$. By this method, we find an upper limit $\delta_{e}<0.175 \%$ at $3-\sigma$. This value is larger than in the case of pure white noise because it accounts for correlated noise. We also observed this system at the predicted time of an eclipse with the $36 \mathrm{~cm}$ telescope. We did not detect the eclipse, but the limit on $\delta_{e}$ obtained from these observations is less constraining than that obtained from the phase-folded XO lightcurve. Overall, we do not find any sign that would indicate a stellar eclipsing binary or triple star system.

\subsection{Radial velocity follow-up}

Radial velocity follow-up was conducted between September 2013 and January 2016 with the SOPHIE spectrograph (Bouchy et al. 2009) at the Observatoire de Haute-Provence, France (Table 3). Exposure times of a few minutes allowed signal-to-noise ratios per pixel at $550 \mathrm{~nm}$ around 45 to be reached on most of the 


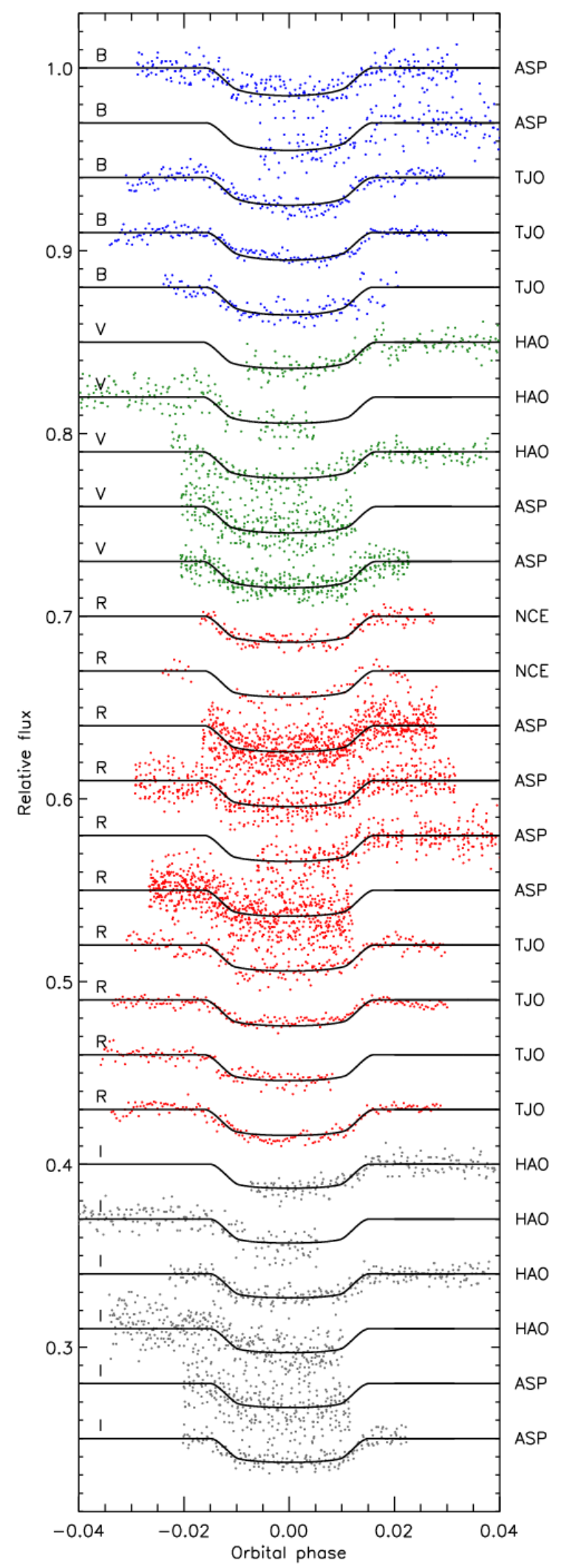

Figure 2. Photometric follow-up observations of $\mathrm{XO}-6 \mathrm{~b}$ for individual transits and bandpasses. The bandpasses are noted as $\mathrm{B}$ (blue), V (green), R (red), and I (grey), and the observatories are labeled as in Table 1. Only lightcurves of good quality are shown. The best transit model calculated for each bandpass is overplotted as a black line. The different lightcurves do not have the same time sampling, therefore the apparent point-to-point dispersion is not representative of their relative quality. Lightcurves are offset for clarity.

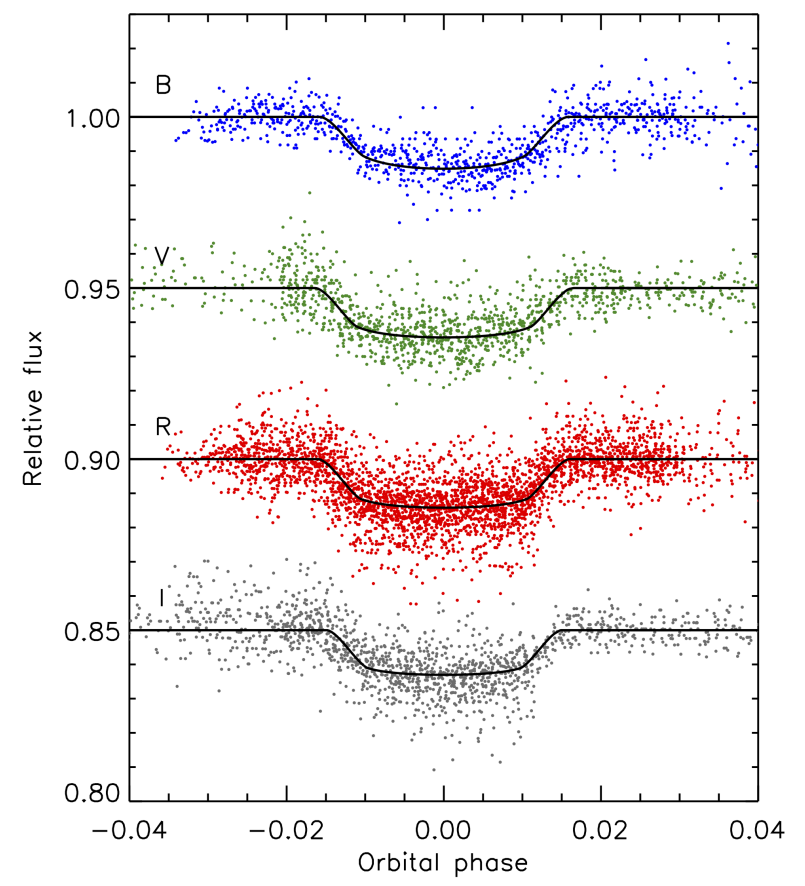

Figure 3. Photometric follow-up observations of XO-6b in several bandpasses: B (blue), V (green), R (red), and I (grey) from top to bottom. Data from different observations are blended in their respective filter bands. The best transit model in each bandpass is overplotted as a black line. Lightcurves are offset for clarity.

exposures. We used the SOPHIE pipeline to extract the spectra from the detector images, cross-correlate them with a G2-type numerical mask which produced clear cross-correlation functions (CCFs), then fit the CCFs by Gaussians to get the radial velocities (Baranne et al. 1996: Pepe et al. 2002). Measurements obtained along the orbit show that $\mathrm{XO}-6$ is a single, fast rotating star with $v \sin i_{\star} \approx 45 \mathrm{~km} \mathrm{~s}^{-1}$. The large width of the spectral lines implies a moderate accuracy of the radial velocity measurements, of the order of $\pm 90 \mathrm{~m} \mathrm{~s}^{-1}$. The phase-folded radial velocities secured out of the transit are plotted in Figure 4. They show a hint for a detection of the reflex motion of the star due to its planet. The fit of these radial velocities with a Keplerian, circular orbit phased according to the transits provides a semi-amplitude $K=200 \pm 70 \mathrm{~m} \mathrm{~s}^{-1}$, corresponding to a planetary mass $M_{p}=1.9 \pm 0.5 \mathrm{M}_{\mathrm{Jup}}$. The dispersion of the residuals is $150 \mathrm{~m} \mathrm{~s}^{-1}$, slightly above the expected error bars on radial velocities, which indicates that systematic effects remain. We rather adopt the 3- $\sigma$ upper limit for its detection, $K<450 \mathrm{~m} \mathrm{~s}^{-1}$, corresponding to a planetary mass $M_{p}<4.4 \mathrm{M}_{\text {Jup }}$. Many additional radial velocity measurements would be required for a significant measurement of the planetary mass.

We observed XO-6 with SOPHIE during a transit in order to detect and characterize the planet through the analysis of the Rossiter-McLaughlin effect. The first 6 attempts were canceled due to bad weather. Such observations require excellent weather conditions for much of a night, and a 7th attempt was made on January 17, 2015 and was successful: the transit was observed in good conditions yielding 23 measurements with signalto-noise ratios similar as above. We clearly detect the Rossiter-McLaughlin anomaly which confirms the exis- 
Table 3

Radial velocities of XO-6 measured along the orbit and during the transit of January 17, 2015 using the SOPHIE spectrograph at the Observatoire de Haute-Provence.

\begin{tabular}{cccc}
\hline $\begin{array}{c}\text { Reduced } \\
\text { BJD }\end{array}$ & $\begin{array}{c}\text { Orbital } \\
\text { phase }\end{array}$ & $\begin{array}{c}\text { RV } \\
{\left[\mathrm{km} \mathrm{s}^{-1}\right]}\end{array}$ & $\begin{array}{c}1-\sigma \text { uncertainty } \\
{\left[\mathrm{km} \mathrm{s}^{-1}\right]}\end{array}$ \\
\hline 56551.6553 & 0.1588 & -5.965 & 0.109 \\
56560.6592 & -0.4497 & -5.394 & 0.114 \\
56560.6617 & -0.4491 & -5.574 & 0.100 \\
56723.3858 & -0.2289 & -5.090 & 0.089 \\
56974.5451 & 0.4801 & -5.642 & 0.090 \\
57040.3717 & -0.0361 & -5.236 & 0.089 \\
57040.3814 & -0.0335 & -5.143 & 0.085 \\
57040.3916 & -0.0308 & -5.265 & 0.080 \\
57040.4017 & -0.0281 & -5.153 & 0.083 \\
57040.4114 & -0.0255 & -5.252 & 0.081 \\
57040.4209 & -0.0230 & -5.109 & 0.086 \\
57040.4300 & -0.0206 & -5.196 & 0.089 \\
57040.4390 & -0.0182 & -5.279 & 0.085 \\
57040.4486 & -0.0156 & -5.182 & 0.087 \\
57040.4585 & -0.0130 & -5.034 & 0.087 \\
57040.4683 & -0.0104 & -4.958 & 0.087 \\
57040.4784 & -0.0077 & -5.171 & 0.089 \\
57040.4885 & -0.0051 & -5.241 & 0.095 \\
57040.4986 & -0.0024 & -5.521 & 0.087 \\
57040.5085 & 0.0003 & -5.473 & 0.086 \\
57040.5181 & 0.0028 & -5.652 & 0.086 \\
57040.5272 & 0.0052 & -5.706 & 0.083 \\
57040.5364 & 0.0077 & -5.664 & 0.090 \\
57040.5460 & 0.0102 & -5.533 & 0.089 \\
57040.5561 & 0.0129 & -5.691 & 0.089 \\
57040.5657 & 0.0155 & -5.533 & 0.079 \\
57040.5746 & 0.0178 & -5.239 & 0.088 \\
57040.5840 & 0.0203 & -5.420 & 0.096 \\
57363.6254 & -0.1785 & -5.428 & 0.087 \\
57378.4922 & -0.2298 & -5.249 & 0.080 \\
57383.4730 & 0.0931 & -5.554 & 0.094 \\
57384.5389 & 0.3762 & -5.315 & 0.083 \\
57399.3742 & 0.3165 & -5.603 & 0.112 \\
57402.3977 & 0.1196 & -5.663 & 0.083 \\
57402.6758 & 0.1934 & -5.576 & 0.099 \\
57405.5191 & -0.0514 & -5.426 & 0.109 \\
\hline \hline & & & \\
& &
\end{tabular}

Note. The orbital phase is 0 at mid-transit.

tence of a transiting object in front of the fast rotating star (Figure 5). The amplitude is $\sim 500 \mathrm{~m} \mathrm{~s}^{-1}$ and the variations suggest a prograde, misaligned planetary orbit. We note that the systemic radial velocity is different by about $200 \mathrm{~ms}^{-1}$ between the Keplerian fit of radial velocities secured over more than two years (Figure 4) and the spectral transit observed during a single night (Figure 5). This shift is of the order of magnitude of the Keplerian fit residuals dispersion so it is not significant. This shows that the systematics and stellar jitter are smaller on a few-hour timescale than on a twoyear time scale.

\subsection{Doppler tomography analysis}

We analyzed the spectra obtained during the transit using a line-profile tomography technique as described in Collier Cameron et al. (2010a). This technique is particularly well suited to fast rotators (Collier Cameron et al. 2010b; Bourrier et al. 2015). The planet partial occultation of the star produces a Gaussian "bump" in the stellar line profile, which spectral location depends on

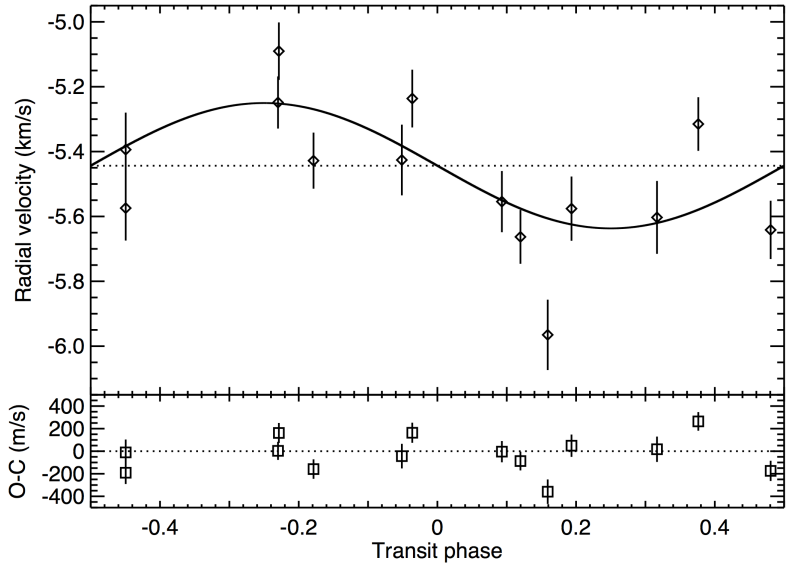

Figure 4. SOPHIE radial velocities of XO-6 with 1- $\sigma$ error bars, phase-folded with the period $P=3.765$ days. A Keplerian, circular fit is overplotted as a plain line. There is a hint of detection, with a semi-amplitude $K=200 \pm 70 \mathrm{~m} \mathrm{~s}^{-1}$ corresponding to a planet mass $M_{p}=1.9 \pm 0.5 \mathrm{M}_{\mathrm{Jup}}\left(K<450 \mathrm{~m} \mathrm{~s}^{-1}\right.$ at $\left.3 \sigma\right)$. The bottom panel shows the residuals of that fit.

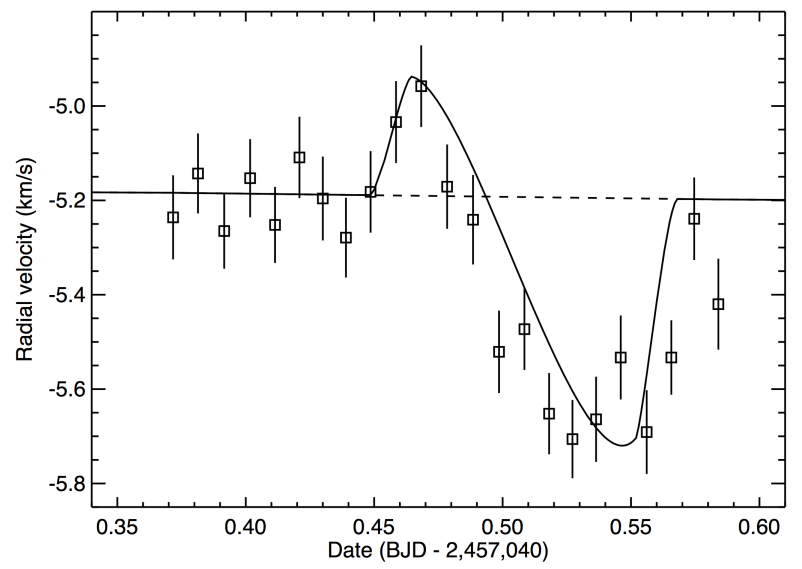

Figure 5. SOPHIE radial velocities of XO-6 with 1- $\sigma$ error bars, secured during the January 17, 2015 transit. The RossiterMcLaughlin anomaly is clearly detected. Overplotted is the model corresponding to the parameters measured through Doppler tomography analysis (Section 4.4 .

the planet position in front of the stellar disk during the transit (Figure 6). We fit the CCFs of the stellar spectra with a transit model, with and without prior constraints obtained previously from the photometric transits.

We use the spectra before and after the transit to measure the fixed pattern in the CCF (the so-called "side-lobes pattern", Collier Cameron et al. 2010a), which is assumed to be constant over the same night and are only function of the velocity point $j$ in the CCF abscissa. The fixed pattern is first estimated by the mean of the residual in the out-of-transit spectra after a fit to the data by the model of the stellar line. We then simultaneously search for the fixed pattern and the best star+transit model in a global fit. The free parameters of the fit are the systemic velocity $v_{\star}$, the star projected rotational velocity $v \sin i_{\star}$, the system size $a / R_{\star}$, the planet-to-star size ratio $R_{p} / R_{\star}$, the inclination of the planetary orbit $i$, the sky-projected obliquity $\lambda$, and the local line profile width $s=\sigma /\left(v \sin i_{\star}\right)$ expressed in units of the projected stellar rotational ve- 
locity, where $\sigma=F W H M /(2 \sqrt{2 \ln 2})$ and $F W H M$ is the full-width at half-maximum of the non-rotating stellar line profile convolved with the instrument profile (see details in Bourrier et al. 2015). The orbital period and the central time of the transit are taken from the analysis of the photometric data. We performed a fit with and without the constraints on the two free parameters measured independently from the photometric data $\left(a / R_{\star}\right.$ and $\left.i\right)$. The merit function of the fit is a $\chi^{2}$ function as in Bourrier et al. (2015):

$$
\begin{aligned}
\chi^{2}= & \sum_{i}^{n_{C C F}} \sum_{j}^{n_{v}}\left[\frac{f_{i, j}(\text { model })-f_{i, j}(\text { obs })}{\sigma_{i}}\right]^{2}+ \\
& \sum_{a_{\mathrm{p}} / R_{\star}, i_{\mathrm{p}}}\left[\frac{x_{\text {tomo }}-x_{\text {photo }}}{\sigma_{x_{\text {photo }}}}\right]^{2}+\left(\frac{v_{\star}-v_{\star, o b s}}{\sigma_{v_{\star}, o b s}}\right)^{2},
\end{aligned}
$$

where $f_{i, j}$ is the flux at velocity point $j$ in the $i$ th observed or model CCFs. The error on the flux, $\sigma_{i}$, is supposed constant for a given CCF. The photometric measurements of the semi-major axis and orbital inclination $\left(x_{\text {photo }} \pm \sigma_{x_{\text {photo }}}\right)$ can be used to constrain the model values of these parameters $x_{\text {tomo }}$ (Table 4). The observed star systemic velocity $v_{\star, o b s}$ is taken to be $-5.1 \mathrm{~km} / \mathrm{s}$ with a conservative error bar of $\sigma_{v_{\star}, o b s}=1.0 \mathrm{~km} / \mathrm{s}$. The difference with the systemic velocities reported in Section 4.3 is due to the distinct methods used to determine them from the CCF fits (a simple Gaussian fit in Section 4.3 and a more elaborate profile fit here). These differences have no effects on the results.

For the tabulated errors on the CCF profiles $\sigma_{i}$, we use the residuals between the CCFs and the first best-fit model profile obtained assuming the same error bars for all CCF. We also took into account that the CCFs are calculated by the instrument pipeline at a velocity resolution of $0.5 \mathrm{~km} \mathrm{~s}^{-1}$, while the spectra have an instrumental resolution of about $7.5 \mathrm{~km} \mathrm{~s}^{-1}$. The residuals are thus strongly correlated, which can lead to an underestimation of the error bars on the derived parameters. As in Bourrier et al. (2015) we retrieved the uncorrelated Gaussian component of the CCFs noise (the "white noise") using an analysis of the residuals variance as a function of a data binning size $n_{b i n}$. The variance well matches the quartic harmonic combination of a white and a red noise components:

$$
\sigma^{2}\left(n_{\text {bin }}\right)=\left(\left(\frac{n_{\text {bin }}}{\sigma_{\text {Uncorr }}^{2}}\right)^{2}+\left(\frac{1}{\sigma_{\text {Corr }}^{2}}\right)^{2}\right)^{-\frac{1}{2}} .
$$

where $\sigma_{U n c o r r} / \sqrt{n_{\text {bin }}}$ can be understood as the result of the intrinsic uncorrelated noise after the binning of $n_{\text {bin }}$ pixels, and $\sigma_{C o r r}$ is a constant term characterizing the correlation between the binned pixels. We find that the uncorrelated noise is about $\sqrt{2}$ larger than the dispersion of the non-binned data. For the final fit, we did not bin the data, and for all CCF pixels we use the uncorrelated noise for the tabulated errors $\sigma_{i}$.

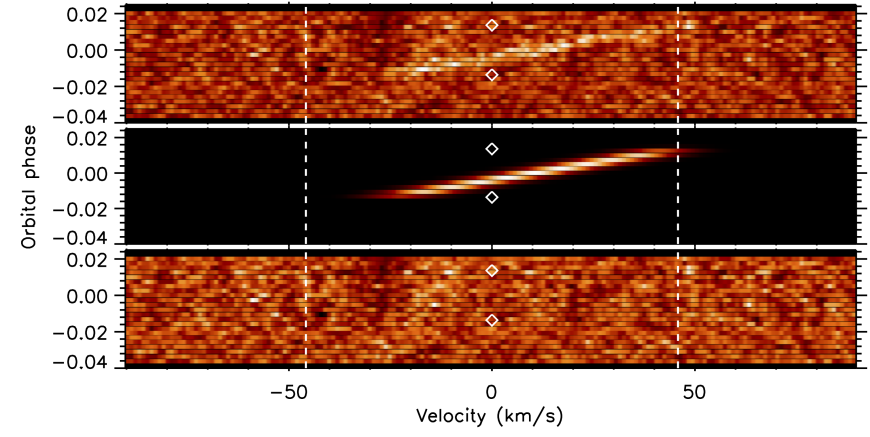

Figure 6. Maps of the time-series CCFs during the transit of January 17,2015 , as a function of the radial velocity relative to the star (in abscissa) and the orbital phase (in ordinate, increasing vertically). Flux values increase from dark red to white. Vertical dashed white lines are plotted at $\pm v \sin i_{\star}$, and the white diamonds indicate the time of the first and the fourth contacts. Top panel: Map of the transit residuals after subtracting the modeled outof-transit CCF. The signature of $\mathrm{XO}-6 \mathrm{~b}$ is a bright and wide feature that crosses the line profile from $\sim-20 \mathrm{~km} \mathrm{~s}^{-1}$ at the ingress to $\sim+40 \mathrm{~km} \mathrm{~s}^{-1}$ at the egress. The dark feature at $\mathbf{- 3 0} \mathrm{km} \mathrm{s}^{-1}$ probably arises from a stellar pulsation. Middle panel: Bestfit model for the XO-6b transiting signature. The parameters of this best-fit model are constrained by both the spectroscopic and the photometric data. Bottom panel: Overall residual map after the subtraction of the model planet signature.

The result of the fit to the spectroscopic profiles of the transit is summarized in Table 4. The $v \sin i_{\star}$ is measured to be $48 \pm 3 \mathrm{~km} \mathrm{~s}^{-1}$. The transit of the planet is clearly detected in the CCF profiles (Figure 6). The effect of the dark silhouette of the planet is a bump superimposed on the stellar absorption line, which goes from about $-20 \mathrm{~km} \mathrm{~s}^{-1}$ at the ingress, to about $+40 \mathrm{~km} \mathrm{~s}^{-1}$ at the egress, both relative to the systemic velocity of the star. This asymmetry is a signature of a prograde orbit that is misaligned to the equatorial plane of the star: the ingress of the planet shadow is at larger latitude on the stellar disk than the egress. The spectroscopic detection of the planet shadow yields an estimate of the planet size $\left(R_{p} / R_{\star}=0.107_{-0.005}^{+0.006}\right)$, which is totally independent from and consistent with the estimate from time-series photometry. This shows that the transiting object detected by the tomographic analysis and from the photometric lightcurves is indeed the same, and indeed transits in front of XO-6. The combined measurements using both photometric estimate and spectroscopy data gives $R_{p} / R_{\star}=0.110 \pm 0.006$.

Finally, the planet orbit misalignment is robustly established by the asymmetry of the Rossiter-MacLaughin radial velocity anomaly seen in the radial velocity curve during the transit (Figure 5) and the Doppler position of the planet shadow on the stellar disk seen in the CCF profiles (Figure 6). We obtain a measurement of the sky-projected obliquity that is at 9- $\sigma$ different from zero: $\lambda=-20.7 \pm 2.3$ degrees.

\subsection{The planetary nature of $X O-6 b$}

Because of the fast stellar rotation, the recoil radial velocity amplitude and mass determination of XO-6b are uncertain. In the following, we review the possible configurations for the XO-6 system and assess the planetary nature of the transiting object through the elimination process, as done for KOI-12b (Kepler-448b) (Bourrier et al. 2015$)$.

- The radial velocity measurements taken during the 
Table 4

Results of the Doppler tomography, with and without the constraints from the photometric data. The $3^{\text {rd }}$ column reports the values obtained with the analysis of the photometric data only. The $4^{\text {th }}$ column, labeled Tomography, gives the results obtained using the spectroscopic data only. The $5^{\text {th }}$ column gives the results obtained using both the fit to the spectroscopic data and the additional constraints of the two first values (scaled semi-major axis and orbital inclination) from the photometry as tabulated in the $3^{\text {rd }}$ column.

\begin{tabular}{lccccl}
\hline Parameter & Symbol & Photometry & Tomography & $\begin{array}{c}\text { Tomography } \\
+ \text { Photometry }\end{array}$ & Unit \\
\hline \hline Scaled semi-major axis & $a / R_{\star}$ & $9.20 \pm 0.19$ & $8.3_{-0.8}^{+1.2}$ & $9.08 \pm 0.17$ & - \\
Orbital inclination & $i$ & $85.95 \pm 0.20$ & $85.4_{-1.0}^{+1.5}$ & $86.0 \pm 0.2$ & $\mathrm{deg}$ \\
Planet-to-star radii ratio & $R_{\mathrm{p}} / R_{\star}$ & $0.1144 \pm 0.0013$ & $0.107_{-0.005}^{+0.006}$ & $0.110 \pm 0.006$ & - \\
Stellar rotation velocity & $v \sin i_{\star}$ & - & $51 \pm 5$ & $48 \pm 3$ & $\mathrm{~km} \mathrm{~s}^{-1}$ \\
Local line width ratio & $s^{\dagger}$ & - & $0.120_{-0.013}^{+0.016}$ & $0.129 \pm 0.013$ & - \\
Local line width & $s \times v \sin i_{\star}$ & - & $6.1 \pm 0.3$ & $6.2_{-0.4}^{+0.5}$ & $\mathrm{~km} \mathrm{~s}^{-1}$ \\
Sky-projected obliquity & $\lambda$ & - & $-18.1_{-8.0}^{+3.4}$ & $-20.7 \pm 2.3$ & $\mathrm{deg}$ \\
\hline \hline
\end{tabular}

$\dagger: s=\mathrm{FWHM} /\left(2 \sqrt{2 \ln 2} v \sin i_{\star}\right)$.

orbit provide a 3- $\sigma$ upper limit for the mass of the companion of $M_{p}<4.4 \mathrm{M}_{\mathrm{Jup}}$. Therefore, we can exclude the case of an unblended stellar eclipsing binary system.

- An eclipsing binary in the background of XO-6 could mimic the photometric transit signatures. However, the detection of the Rossiter-McLaughlin effect and the tomographic analysis indicate that the transit occurs in front of the fast rotating star, which rules out a background eclipsing binary as the source of the transits.

- If XO-6 is an eclipsing binary system diluted in the light of third star, this star might contaminate the transit signatures and stellar line profile distortions. However, no indication of another star is seen in the spectra. If such a star is present but too faint to be detected, then it would not affect significantly the analysis nor the planetary nature of XO-6b. In addition, the transit parameters extracted from the photometry and from the tomographic analysis are consistent with each other. This argues against the case of a third star that would contaminate the photometric transit signature without affecting the spectroscopic measurements, and vice-versa. If a third star is present and bright enough to affect all measurements consistently, it should have the same spectral type, same systemic radial velocity, same rotation rate, and same spin alignment as XO-6 to remain undetected in the spectra and in the tomographic analysis; this contrived identical twin stars configuration is extremely improbable.

Overall, the ensemble of data allows us to validate the planetary nature of XO-6b. Its parameters are given in Table 5 .

\section{STELLAR PARAMETERS}

We derive the stellar properties from a spectral analysis of SOPHIE spectra. We keep only spectra with a signal to noise ratio larger than 40 , and co-add them once corrected from the radial velocities and set in the rest frame. The final spectrum has a signal to noise ratio of $\sim 640$ per resolution element in the continuum at
Table 5

Parameters of the XO-6 system assuming a circular orbit.

\begin{tabular}{cccc}
\hline \hline Parameter & Units & Value & $1-\sigma$ uncertainty \\
\hline$P$ & {$[\mathrm{days}]$} & 3.7650007 & 0.0000081 \\
$T_{0}$ & {$[\mathrm{BJD}]$} & 2456652.71245 & 0.00055 \\
$a / R_{\star}$ & & 9.08 & 0.17 \\
$R_{p} / R_{\star}$ & & 0.110 & 0.006 \\
$i$ & {$[\mathrm{deg}]$} & 86.0 & 0.2 \\
$\tau_{14}$ & {$[$ hours $]$} & 2.90 & 0.10 \\
$\tau_{23}$ & {$[$ hours $]$} & 1.99 & 0.12 \\
$b$ & & 0.633 & 0.034 \\
$a$ & {$[\mathrm{AU}]$} & 0.0815 & 0.0077 \\
$\rho_{\star a}{ }^{\dagger}$ & {$\left[\mathrm{g} \mathrm{cm}^{-3}\right]$} & 1.0 & 0.056 \\
$K_{\star}$ & {$\left[\mathrm{m} \mathrm{s}^{-1}\right]$} & 200 & 70 \\
& & $<450$ & \\
$M_{p}$ & {$[\mathrm{M} \mathrm{Jup}]$} & 1.9 & 0.5 \\
$R_{p}{ }^{\dagger}$ & {$\left[\mathrm{R}_{\mathrm{Jup}}\right]$} & $<4.4$ & \\
$\rho_{p}$ & {$\left[\mathrm{~g} \mathrm{~cm}^{-3}\right]$} & 0.27 & 0.22 \\
& & $<0.62$ & 0.11 \\
$g_{p}$ & {$\left[\mathrm{~m} \mathrm{~s}^{-2}\right]$} & 26.4 & 9.7 \\
& & $<59.4$ & 80 \\
$H$ & {$[\mathrm{~km}]$} & 216 & 28 \\
$T_{e q}$ & {$[\mathrm{~K}]$} & $>96$ & \\
\hline \hline
\end{tabular}

Notes. ${ }^{\dagger}$ See Section 6.1 for a discussion on the planetary radius and the stellar density. $<$ indicate 3- $\sigma$ upper limits, $>$ indicate 3- $\sigma$ lower limits.

$5610 \AA$. However, the fast stellar rotation yields wide and blended spectral lines which complicate the analysis. First, we derive $v \sin i_{\star}$ and the macroscopic velocity $v_{\text {macro }}$ by analyzing isolated spectral lines in the Fourier space. Then, we use the SME package (Valenti \& Piskunov 1996) to fit the observed spectrum to a synthetic spectrum. We use reference abundances from Asplund et al. (2005). We derive the effective temperature $T_{\text {eff }}$ on the hydrogen lines, the gravity $\log g_{\star}$ on the $\mathrm{Ca}$ $\mathrm{I}$ and $\mathrm{Mg} \mathrm{I}$ lines, and the metallicity $[\mathrm{Fe} / \mathrm{H}]$, all of which through an iterative process. The results are reported in Table 6. The values for $T_{e f f \star}$ and $[F e / H]$ are compatible within their uncertainties with those reported by Ammons et al. (2006). Using the atmospheric parameters $T_{e f f \star}, \log g_{\star}$, and $[\mathrm{Fe} / \mathrm{H}]$ from the spectral analysis, we estimate the mass, radius, and age by a MCMC minimisation procedure using the STAREVOL stellar evolution 
models (Siess et al. 2000; Palacios et al. 2003, 2006, Decressin et al. 2009, Lagarde et al.2012). 'The atmospheric parameters are consistent with main sequence evolutionary tracks, and marginally consistent with pre-main sequence tracks. We exclude the pre-main sequence cases because the star does not show any sign of a young age. We also exclude the post-main sequence cases as they are outside of the $1-\sigma$ uncertainties of $T_{e f f \star}$ and $\log g_{\star}$. Finally, our models do not include overshooting, but for XO-6 the major source of uncertainty arises from the stellar rotation. The posterior distributions for the stellar age, mass, and radius are shown in Figure 7 and the corresponding parameters are reported in Table 6 . The distributions are bimodal for the age and mass; we also report in Table 6 the solutions that correspond to these two modes taken individually (solutions a and b). Using the stellar density obtained from the photometric $a / R_{\star}$ instead of the spectroscopic $\log g_{\star}$ would yield different solutions for the stellar age, mass, and radius. However, because the orbital solution is poorly constrained, we keep $\log g_{\star}$ as an input for the stellar evolution models (see Section 6.1 for a discussion on the stellar density).

Table 6

Properties of the XO-6 star.

\begin{tabular}{cccc}
\hline \hline Quantity & Unit & Value & Notes \\
\hline Name & & TYC 4357-995-1 & 1 \\
RA & J2000 & $06: 19: 10.3604$ & 1 \\
DEC & J2000 & $+73: 49: 39.602$ & 1 \\
V & Mag & $10.25 \pm 0.03$ & 2 \\
$\mathrm{H}$ & $\mathrm{Mag}$ & $9.266 \pm 0.017$ & 2 \\
$\mathrm{~B}-\mathrm{V}$ & $\mathrm{Mag}$ & $0.43 \pm 0.04$ & 2 \\
Distance & $\mathrm{pc}$ & $86_{-31}^{+79}$ & 3 \\
& & 196 & 4 \\
Spectral type & & $\mathrm{F} 5$ & 2,5 \\
$T_{\text {ef } f \star}$ & $\mathrm{K}$ & $6720.00 \pm 100$ & 5 \\
{$[F e / H]$} & & $-0.07 \pm 0.1$ & 5 \\
log $g_{\star}$ & & $4.04 \pm 0.10$ & 5 \\
$v_{\text {micro }}$ & $\mathrm{km} \mathrm{s}^{-1}$ & $1.95 \pm 0.5$ & 5 \\
$v_{\text {macro }}$ & $\mathrm{km} \mathrm{s}-1$ & $20.6 \pm 4.5$ & 5 \\
$v$ sin $i_{\star}$ & $\mathrm{km} \mathrm{s}-1$ & $44.2 \pm 3.0$ & 5 \\
Mass & $\mathrm{M}_{\odot}$ & $1.47 \pm 0.06$ & 5 \\
Radius & $\mathrm{R}_{\odot}$ & $1.93 \pm 0.18$ & 5 \\
Age & $\mathrm{Gyr}$ & $1.88_{-0.20}^{+0.90}$ & 5 \\
\hline Mass & $\mathrm{M}_{\odot}$ & $1.50 \pm 0.03$ & $5, \mathrm{a}$ \\
Radius & $\mathrm{R}_{\odot}$ & $1.86 \pm 0.16$ & $5, \mathrm{a}$ \\
Age & $\mathrm{Gyr}$ & $1.86 \pm 0.20$ & $5, \mathrm{a}$ \\
Mass & $\mathrm{M} \odot$ & $1.39 \pm 0.04$ & $5, \mathrm{~b}$ \\
Radius & $\mathrm{R}_{\odot}$ & $2.05 \pm 0.12$ & $5, \mathrm{~b}$ \\
Age & $\mathrm{Gyr}$ & $2.74 \pm 0.32$ & $5, \mathrm{~b}$ \\
\hline \hline
\end{tabular}

Notes. 1: Hog et al. (1998). 2: Høg et al. (2000). 3: Ammons et al. (2006). 4: Pickles \& Depagne (2010). 5: This work. a, b: Solutions tor each of the two modes (see text and Figure 7).

\section{DISCUSSION}

\subsection{Properties of the hot Jupiter XO-6b}

The orbits of hot Jupiters are expected to be circularized (Leconte et al. 2010). However, several hot Jupiters have been found on eccentric orbits such as XO-3b $(e=0.26)$, HAT-P-34b $(e=0.44)$, HAT-P-2b $(e=0.52)$, or CoRoT-20b $(e=0.56)$. In the case of XO-6, the stellar rotation may excite the planetary orbit
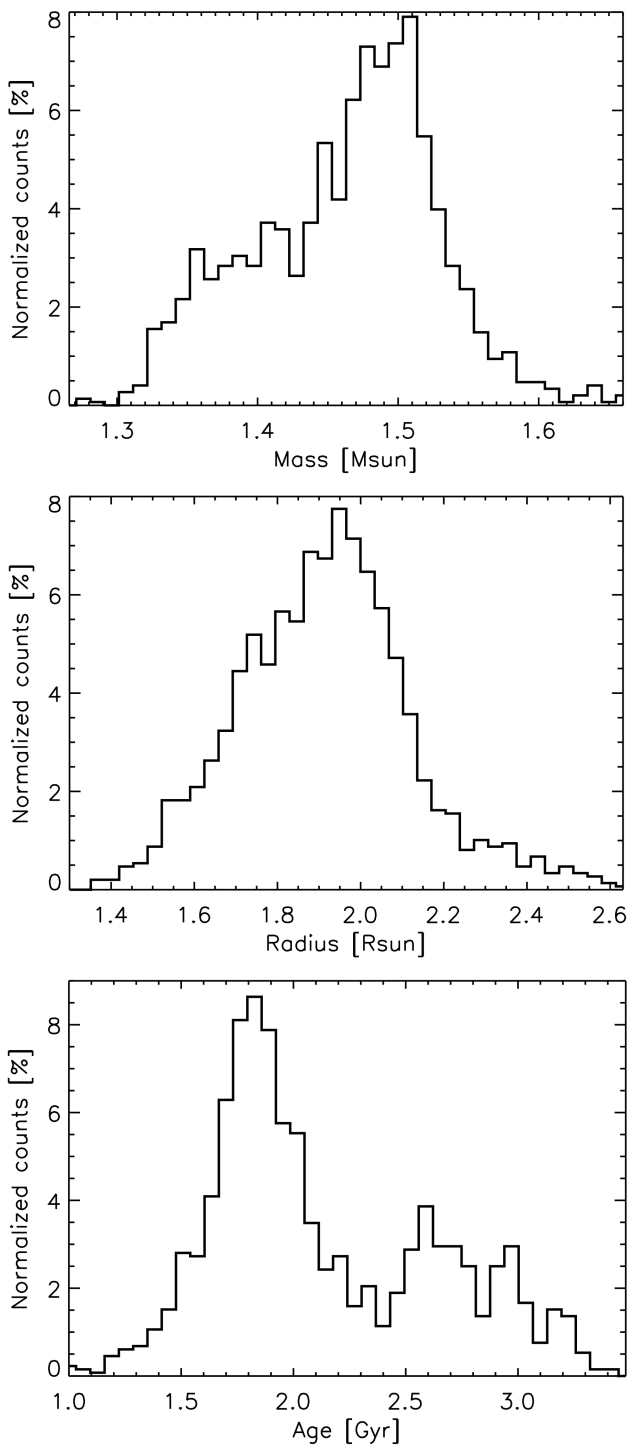

Figure 7. Distributions of the mass, radius, and age for the XO-6 star.

and prevent its circularization, as discussed in Section 6.3 However, without a constraint on the eccentricity due to the difficulty of measuring radial velocities on this fast rotating star, we derive the planet parameters assuming a circular orbit. Using the stellar radius derived in Section 5 we find a planetary radius $R_{p}=2.07 \mathrm{R}_{\text {Jup }}$. Assuming a mass $M_{p}=1.9 \mathrm{M}_{\text {Jup }}$ suggested by the radial velocities and a resulting density $\rho_{p}=0.27 \mathrm{~g} \mathrm{~cm}^{-3}$, we find that XO-6b is extremely inflated compared to model predictions for hot Jupiters. Based on models by Guillot et al. (2006, see also Moutou et al. 2013), XO-6b appears to have a positive radius anomaly of about $+65000 \mathrm{~km}$ that is the largest of known exoplanets. For comparison, another extremely inflated planet, CoRoT-2b, has a radius anomaly of about $+30000 \mathrm{~km}$ which already cannot be explained by standard recipes (e.g. Guillot \& Havel 2011). However, this extremely large $R_{p}$ value might be due to a poor estimate of $R_{\star}$. Using $\rho_{\star a}$ instead of $\log g_{\star}$ as an input for the stellar evolution models, the stellar radius would decrease to $1.21 \mathrm{R}_{\odot}$ and would yield a planetary radius of $1.30 \mathrm{R}_{\mathrm{Jup}}$. Although still inflated, 
this would be in line with other inflated hot Jupiters.

Using Equation 4 of Southworth et al. (2007) with $e=0$ and $K_{\star}=200 \mathrm{~m} \mathrm{~s}^{-1}$, we derive a planet's surface gravity $g_{p}=26 \mathrm{~m} \mathrm{~s}^{-2}$. Assuming a zero albedo, we derive an equilibrium temperature $T_{e q}=1577 \mathrm{~K}$ and a scale height $H=216 \mathrm{~km}$. This temperature is close to the condensation temperature of species thought to be responsible for clouds in the atmospheres of hot Jupiters, such as $\mathrm{MgSiO}_{3}$ (Lodders 1999 : Lecavelier Des Etangs et al. 2008: Pont et al. 2013). XO-6b is warm compared to other close-in gas giant planets for which molecular spectral signatures have been detected, such as HD 209458b, XO-1b, and HD 189733b (Deming et al. 2013, McCullough et al. 2014. Crouzet et al. 2014), and it orbits a bright star $(\mathrm{H}=9.27)$. In addition, the nearby star located at only 38" separation and only 1 magnitude fainter may provide an excellent reference. Thus, XO-6b is well suited to atmospheric studies, and the XO-6 system is untypical because of the high temperature of the host star.

Because the star is a fast rotator, measuring the mass of $\mathrm{XO}-6 \mathrm{~b}$ is challenging. A few other transiting hot Jupiters have been found around such hot and fast rotating stars (Table 7). CoRoT-11, WASP-33, and KELT-7 have been observed extensively in radial velocities, with 31,248 , and 36 measurements along the orbit respectively, yielding the planets' masses (Gandolfi et al. 2010 Lehmann et al. 2015, Bieryla et al. 2015). CoRoT-3b is so massive that the recoil motion overwhelms stellar rotation effects (Deleuil et al. 2008). Only a mass upper limit has been inferred for KOI-12 (Bourrier et al. 2015) . Kepler-13Ab has its mass determined from the Kepler photometric lightcurves (Shporer et al. 2011; Mazeh et al. 2012, Mislis \& Hodgkin 2012 Shporer et al. 2014, see also Santerne et al. (2012)). For XO-6, we collected 14 spectra along the orbit and we detect a hint of variation (Figure 4); an extensive radial velocity campaign would be necessary to measure the planet's mass with greater confidence. An alternative approach would be to derive the mass from spectroscopy using a method suggested by de Wit \& Seager (2013), in which the planet's mass is obtained from the atmospheric Rayleigh scattering slope and the atmospheric temperature:

$$
M_{p}=-\frac{4 k T\left[R_{p}(\lambda)\right]^{2}}{\mu G \frac{\mathrm{d} R_{p}(\lambda)}{\mathrm{d} \ln \lambda}}
$$

where $k$ is the Boltzmann constant, $T$ the planet's atmospheric temperature, $\mu$ the atmospheric mean molecular mass, $G$ the gravitational constant, and $\lambda$ the wavelength. The Rayleigh scattering slope $\frac{\mathrm{d} R_{p}(\lambda)}{\mathrm{d} \ln \lambda}$ could be obtained by transmission spectroscopy with $H S T$ STIS and ACS, and the atmospheric temperature could be inferred by measuring the eclipse depth with Spitzer. Although expensive in terms of resources, these measurements would also characterize the atmosphere of XO-6b.

The radial velocities do not constrain the eccentricity and we assumed $e=0$ in our analysis. Under this assumption, the stellar density obtained from the photometric transit curve is $\rho_{\star a}=\frac{3 \pi}{G P^{2}}\left(\frac{a}{R_{\star}}\right)^{3}=1 \pm$ $0.056 \mathrm{~g} \mathrm{~cm}^{-3}$. This density is $1-\sigma$ away from the closest evolution track for these $T_{e f f \star}$ and $\mathrm{Fe} / \mathrm{H}$. In addition, it differs significantly from that obtained from the mass
Table 7

Hot Jupiter systems with $v \sin i_{\star}>30 \mathrm{~km} \mathrm{~s}^{-1}$ or $T_{e f f \star}>6700 \mathrm{~K}$, and a measured sky-projected obliquity.

\begin{tabular}{|c|c|c|c|c|}
\hline Name & $\begin{array}{c}T_{e f f \star} \\
{[\mathrm{K}]}\end{array}$ & $\begin{array}{c}v \sin i_{\star} \\
{\left[\mathrm{km} \mathrm{s}^{-1}\right]}\end{array}$ & $\begin{array}{c}\lambda \\
{[\mathrm{deg}]}\end{array}$ & $\begin{array}{c}M_{p} \\
{\left[\mathbf{M}_{\mathrm{Jup}}\right]}\end{array}$ \\
\hline CoRoT-11 & $6440 \pm 120$ & $40 \pm 5$ & $0.1 \pm 2.6$ & $2.33 \pm 0.27^{a}$ \\
\hline XO-6 & $6720 \pm 100$ & $48 \pm 3$ & $\mathbf{- 2 0 . 7} \pm \mathbf{2 . 3}$ & $<4.4^{b}$ \\
\hline CoRoT-3 & $6740 \pm 140$ & $17 \pm 1$ & $-37.6_{-10}^{+22.3}$ & $21.77 \pm 1.0^{c}$ \\
\hline KELT-7 & $6789_{-49}^{+50}$ & $65_{-5.9}^{+6}$ & $9.7 \pm 5.2$ & $1.28 \pm 0.18^{d}$ \\
\hline KOI-12 & $6820 \pm 120$ & $60_{-0.8}^{+0.9}$ & $12.5_{-2.9}^{+3}$ & $<10^{e}$ \\
\hline WASP-33 & $7430 \pm 100$ & $90 \pm 10$ & $-108.8 \pm 1$ & $2.1 \pm 0.2^{f}$ \\
\hline Kepler-13A & $7650 \pm 250$ & $78 \pm 15$ & $23 \pm 4$ & $6.51 \pm 1.57^{g}$ \\
\hline
\end{tabular}

Reference for $M_{n}$ : a: Moutou et al. (2013). b: This work. c: Deleuil et al. (2008); Moutou et al. (2013). d: Bieryla et al. (2015). e: Bourrier et al. (2015). t: Lehmann et al. (2015). g: Shporer et al. (2014).

and radius of Table 6. $\rho_{\star b}=\frac{M_{\star}}{\frac{4}{3} \pi R_{\star}^{3}}=0.29 \pm 0.08 \mathrm{~g} \mathrm{~cm}^{-3}$. This discrepancy may indicate a non-zero eccentricity, which can be estimated by:

$$
\left(\frac{\rho_{\star a}}{\rho_{\star b}}\right)^{1 / 3}=\frac{1+e \sin \omega}{\sqrt{1-e^{2}}}
$$

where $\omega$ is the argument of periastron (Winn 2010 Eq. 29). This yields $e>0.39$ with a true value that depends on the unknown value of $\omega$. The radial velocities are still consistent with such eccentricities. Other hot Jupiters orbiting around fast rotating stars have been found on eccentric orbits, as discussed in Section 6.3. Alternatively, the spectroscopic $\log g_{\star}$ is generally poorly constrained compared to $a / R_{\star}$ (Sozzetti et al. 2007; Torres et al. 2008); thus, the discrepancy in the stellar density might arise from an inaccurate $\log g_{\star}$ as suggested for example in the case of XO-3b (Johns-Krull et al. 2008: Winn et al. 2008). Another possible estimate is to take the closest density to $\rho_{\star a}$ that is allowed by the evolution tracks for these $T_{e f f \star}$ and $\mathrm{Fe} / \mathrm{H}$. This yields $\rho_{\star c}=0.88 \pm 0.07 \mathrm{~g} \mathrm{~cm}^{-3}$, which would imply $e>0.04$. Finally, the discrepancy in $\rho_{\star}$ might be due to a poor estimate of $\mathrm{Fe} / \mathrm{H}$, or to evolution models that are not adapted to this particular star, or to other issues such as stellar spots (e.g. Guillot \& Havel 2011).

\subsection{Obliquity distribution}

$\mathrm{XO}-6 \mathrm{~b}$ adds to the sample of hot Jupiters with a measured sky-projected obliquity. We define the skyprojected obliquity as the angle between the stellar spin axis and the normal to the planet's orbital plane, as in Winn \& Fabrycky (2015). With the rapid growth of such measurements in recent years, obliquities have become a very promising means of distinguishing between different theories for the dynamical history of hot Jupiters: migration through interactions with the protoplanetary disk or disk-free mechanisms involving interactions with a third body or with other planets (e.g. Naoz et al. 2011). In fact, these measurements provide one of the very few observational constraints to these theories. Misalignment of the planet's orbital plane with respect to the stellar spin axis is found to be common: $34 \%$ of known closein gas giant planets have sky-projected obliquities larger than $30^{\circ}$, and $15 \%$ even have a retrograde orbit. A general picture is emerging: planets orbiting stars with rel- 
atively cool photospheres $(T<6100 \mathrm{~K})$ have low obliquities whereas planets orbiting hotter stars show a wide range of obliquities (Winn et al. 2010, Schlaufman 2010; Albrecht et al. 2012, Dawson \& Chiang 2014; Winn \& Fabrycky 2015). The boundary of $6100 \mathrm{~K}$ also coincides with the rotational discontinuity above which stars rotate significantly faster (Kraft 1967). In addition, the highest-mass planets $\left(M_{n}>3 \mathrm{M}_{\mathrm{Jup}}\right)$ seem associated with lower obliquities (Hébrard et al. 2011). As an example of theoretical study, Albrecht et al. (2012) could explain qualitatively the observed obliquity distribution as a consequence of tidal timescales, although more parameters such as wind mass loss, stellar evolution, and magnetic braking may also be involved (Valsecchi \& Rasio 2014).

Almost all stars hosting a transiting hot Jupiter with a measured sky-projected obliquity have a projected rotational velocity $v \sin i_{\star}$ lower than $25 \mathrm{~km} / \mathrm{s}$ (Figure 8 ). $\mathrm{XO}-6$ lies in the hot star region of the obliquity distribution and bridges the gap between slow and fast rotators. Only four systems have greater $T_{e f f \star}$ and $v \sin i_{\star}$ than XO-6: KELT-7, KOI-12, WASP-33, and Kepler-13A ; CoRoT-3 has a $T_{e f f \star}$ similar to XO-6 but rotates much slower and the transiting object is either a very massive planet or a brown dwarf (Table 7). Among the seven systems with $T_{e f f \star}>6700 \mathrm{~K}$ or $v \sin i_{\star}>30 \mathrm{~km} \mathrm{~s}^{-1}$, six of them, including the two most massive planets, have low to moderate sky-projected obliquities ranging from $0^{\circ}$ to $38^{\circ}$ in absolute value $\left(0^{\circ}\right.$ to $25^{\circ}$ if we exclude CoRoT- $\left.3 \mathrm{~b}\right)$. WASP-33b is the only one with a very large sky-projected obliquity and is even in a retrograde orbit. In contrast, for slower rotators between 6100 and $6700 \mathrm{~K}$, the obliquities are widely distributed over the range $\left[0^{\circ}, 180^{\circ}\right]$. We check whether the distribution of the obliquities of planets orbiting stars with $T_{e f f_{\star}}>6700 \mathrm{~K}$ (6 planets) is statistically different from that for stars with $6100<T_{e f f \star}<6700 \mathrm{~K}(29$ planets $)$. We randomly draw $10^{6}$ samples of 6 obliquities from the 29. The fraction (19\%) of samples containing 0 or 1 planet with $|\lambda|>38^{\circ}$ is not small enough to conclude that the $T_{e f f \star}>6700 \mathrm{~K}$ obliquity distribution is statistically different from that of the $6100<T_{e f f \star}<6700 \mathbf{K}$ distribution.

\subsection{Stellar rotation and planetary orbits}

Studies of relations between stellar rotation and planetary orbital motion also yield constraints on the dynamics of hot Jupiters. Using Kepler data, McQuillan et al. (2013) found a dearth of KOIs (Kepler Objects of Interest) at short orbital periods around fast rotating stars: only slow rotators, with rotation periods longer than 5 days, have planets with orbital periods shorter than 3 days. (Teitler \& Königl 2014) attributed this feature to tidal ingestion of close-in planets by their host stars. In addition, several hot Jupiter host stars are in excess rotation, with rotation periods 4 to 8 times smaller than those expected from rotation isochrones, which supports the case for hot Jupiters spinning up their host stars through tidal interactions (Husnoo et al. 2012). A higher magnetic activity has also been found in two hot Jupiter host stars which have excess rotation and for which strong tidal interaction with the planet are expected (Poppenhaeger \& Wolk 2014). A general picture of the dynamical

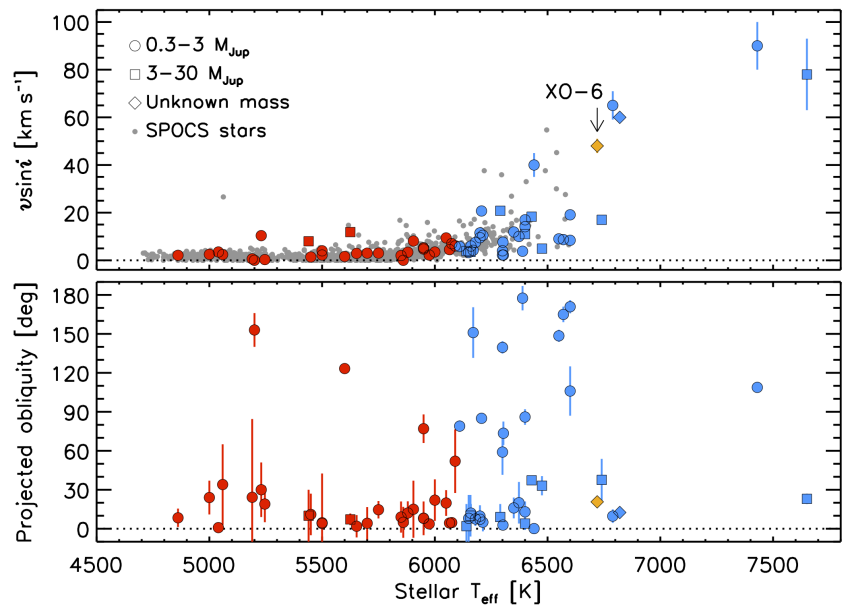

Figure 8. Projected stellar rotation velocity (top) and absolute value of the sky-projected obliquity (bottom) as a function of stellar effective temperature for transiting hot Jupiters $\left(M_{p}>0.3 \mathrm{M}_{\mathrm{Jup}}\right.$, $P<20$ days) with a measured obliquity. The planet mass is encoded by the symbol shape. Color is used to distinguish temperatures below (red) and above (blue) $6100 \mathrm{~K}$. Grey dots are projected rotation rates of stars in the SPOCS catalog (Valenti \& Fischer 2005). The XO-6 system is shown as a yellow diamond shape. Figure adapted from Winn \& Fabrycky (2015), using the databases http://exoplanets.org/ and http://vizier.u-strasbg.fr/

evolution of hot Jupiters and their host stars based on tidal interactions is emerging (Husnoo et al. 2012, Pont 2009: Mazeh et al. 2005). However, this picture applies to the current set of known hot Jupiters, which is almost entirely composed of systems with a stellar rotation period larger than the planet's orbital period $\left(P_{\text {rot }}>P_{\text {orb }}\right)$. In fact, this apparent lack of hot Jupiters around fast rotating stars is intriguing. This may be due to an observational bias: these planets are harder to detect or validate by radial velocities because of stellar line broadening, as illustrated in Section 4.3. and transit searches tend to target cooler stars. Thus, transiting planet candidates with fast rotating host stars may be missed or rejected more readily. XO-6b is one of the very few planets known to orbit a fast rotating star; the stellar rotation period is even smaller than the planet orbital period $\left(P_{\text {rot }}<2.12\right.$ $\left.\mathrm{d}, P_{\text {orb }}=3.77 \mathrm{~d}\right)$ Thus, XO-6 stands in the $P_{\text {rot }}<P_{\text {orb }}$ domain of the $P_{\text {rot }}-P_{\text {orb }}$ diagram (Figure 9), which corresponds to an interaction regime between hot Jupiters and their host stars that is largely unexplored.

For systems with $P_{\text {rot }}<P_{\text {orb }}$, tidal forces would be reversed compared to systems with $P_{\text {rot }}>P_{\text {orb }}$. Tidal forces would raise the planet's angular momentum which would excite its orbit, yielding an increase in orbital period or eccentricity (instead of having the planet spiralling inwards or being circularized on a very close orbit). Over time, tidal dissipation would spin the star down (instead of spinning it up). This tidal push from the star might play a role in the apparent lack of transiting planets at short orbital periods around fast rotators. In this context, it is interesting to note that several hot Jupiters lying in the $P_{\text {rot }}<P_{\text {orb }}$ domain stand out by their large eccentricities: HAT-P-2b $\left(P_{\text {rot }}<3.66 \mathrm{~d}\right.$, $\left.P_{\text {orb }}=5.63 \mathrm{~d}, e=0.52\right)$ and HAT-P-34b $\left(P_{\text {rot }}<2.52\right.$

\footnotetext{
${ }^{4}$ Here, the stellar rotation periods $P_{\text {rot }}$ are estimated by $P_{\text {rot }}=$ $2 \pi R_{\star} / v \sin i_{\star}$ and are therefore upper limits. The true $P_{\text {rot }}$ are smaller by a factor $\sin i_{\star}$, which is usually unknown.
} 
$\left.\mathrm{d}, P_{\text {orb }}=5.45 \mathrm{~d}, e=0.44\right)$ which eccentricities have been inferred from radial velocities, and CoRoT-11b $\left(P_{\text {rot }}<1.73 \mathrm{~d}, P_{\text {orb }}=2.99 \mathrm{~d}, e=0.35\right)$ which eccentricity has been inferred from the timing offset of the secondary eclipse (Parviainen et al. 2013). The other closein gas giant planets with $P_{\text {rot }}<P_{\text {orb }}$ are WASP-33b, OGLE2-TR-L9b, KELT-7b, XO-6b, WASP-7b, each of which do not have a measured eccentricity, Kepler-13Ab which may have a very small but non-zero eccentricity (Shporer et al. 2014), CoRoT-6b $\left(P_{\text {rot }}<6.92 \mathrm{~d}\right.$, $\left.P_{\text {orb }}=8.89 \mathrm{~d}\right)$ which has a negligible eccentricity, and the warm Jupiter KOI-12b (Kepler-448b, $P_{\text {rot }}<1.37 \mathrm{~d}$, $P_{\text {orb }}=17.86 \mathrm{~d}$ ) which does not have a measured eccentricity. Another interesting case is XO-3b which orbits a relatively fast rotator and shows a relatively large eccentricity $\left(P_{\text {rot }}<5.75 \mathrm{~d}, P_{\text {orb }}=3.19 \mathrm{~d}, e=0.29\right)$. Overall, large eccentricities may be common for planets around fast rotators. Such systems may result in a planet orbit - planet spin synchronization around the pericenter, where tidal forces are the strongest, and possibly in stellar spin - planet orbit synchronization also around the pericenter as seems to be the case for CoRoT-11b (Parviainen et al. 2013; Moutou et al. 2013).

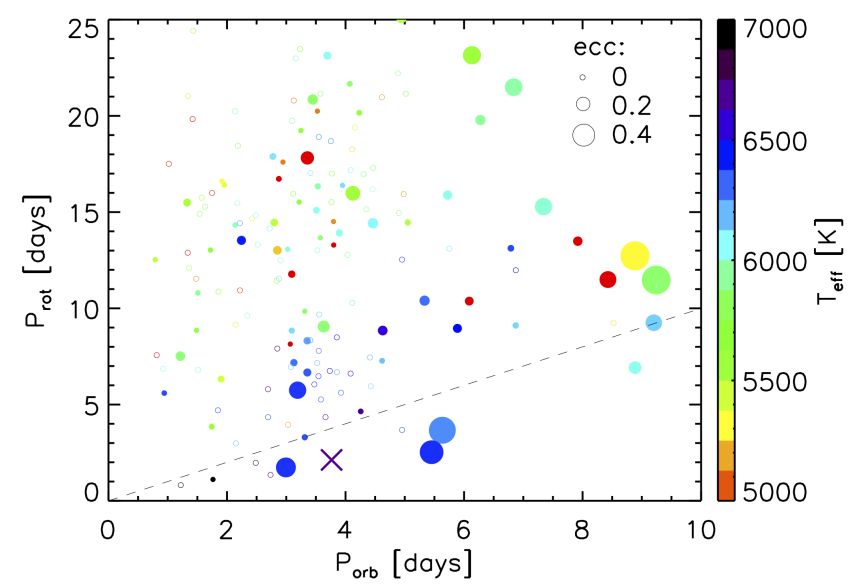

Figure 9. Planetary orbital period $P_{\text {orb }}$ and stellar rotation period $P_{\text {rot }}$ for hot Jupiters $\left(M_{p}>0.3 \mathrm{M}_{\text {Jup }}\right)$. The $P_{\text {rot }}$ are obtained from the stellar $v \sin i_{\star}$ and are therefore upper limits, except in a few cases where they are obtained from photometric modulations. Colors indicate the stellar effective temperature. Sizes represent the orbital eccentricity; planets with no measured eccentricity are represented by open circles. The dashed line shows the $P_{\text {rot }}=P_{\text {orb }}$ synchronization. The XO-6 system is shown as a purple X symbol; its eccentricity is unknown.

\section{CONCLUSION}

We report a transiting hot Jupiter, XO-6b. The fast stellar rotation $\left(v \sin i_{\star}=\mathbf{4 8} \pm \mathbf{3} \mathrm{km} \mathrm{s}^{-1}\right)$ prevents a characterization and mass determination by radial velocities: only a 3- $\sigma$ upper limit was obtained on the planet's mass $\left(M_{p}<4.4 \mathrm{M}_{\mathrm{Jup}}\right)$. We confirm the presence of the transiting object using the Rossiter-McLaughlin effect and we analyze the spectra by Doppler tomography. We show that XO-6b is a transiting hot Jupiter on a prograde, misaligned orbit with a sky-projected obliquity $\lambda=-\mathbf{2 0 . 7} \pm \mathbf{2 . 3}$ degrees. XO-6b orbits around a bright star $(\mathrm{H}=9.27)$ and is relatively warm $\left(T_{e q}=1577 \mathrm{~K}\right)$, thus it is well suited to atmospheric studies. We derive the stellar parameters through a spectral analysis and a minimization in stellar evolutionary tracks. We find that the host star is a F5 star with $T_{e f f \star}=6720 \pm 100$ $\mathrm{K}$ and $[\mathrm{Fe} / \mathrm{H}]=-0.07 \pm 0.1$, and is consistent with the main sequence. We note a discrepancy between the photometric and spectroscopic values of the stellar density, which may indicate an eccentric orbit or may be due to an inaccurate determination of $\log g_{\star}$. This discrepancy also affects the value of the planetary radius. XO-6b adds to the sample of hot Jupiters with a measured skyprojected obliquity, and lies in the hot and fast rotating star region of the obliquity distribution. In addition, the rotation period of the star is smaller than the orbital period of the planet; as a result, tidal forces are reversed compared to hot Jupiters around slow rotators. In this regime, eccentric orbits may be common as suggested by a few striking examples. Overall, the discovery of XO-6b provides a new object to study in the context of dynamical interactions between hot Jupiters and their host stars in a parameter space yet largely unexplored.

NC acknowledges J. Valenti, H. Neilson, and A. H. M. J. Triaud for useful discussions. The Dunlap Institute is funded through an endowment established by the David Dunlap family and the University of Toronto. The XO project is supported by NASA grant NNX10AG30G. AL, VB, GH, and LA have been supported by an award from the Fondation Simone et Cino Del Duca, and acknowledge the support of the French Agence Nationale de la Recherche (ANR), under program ANR-12-BS05-0012 "Exo-Atmos". IR, FV and EH acknowledge support from the Spanish MINECO through grant ESP2014- 57495-C2-2-R. The Joan Oró Telescope (TJO) of the Montsec Astronomical Observatory (OAdM) is owned by the Generalitat de Catalunya and operated by the Institute for Space Studies of Catalonia (IEEC). VB work has been carried out in the frame of the National Centre for Competence in Research "PlanetS" supported by the Swiss National Science Foundation (SNSF). V.B. acknowledges the financial support of the SNSF. This research has made use of the Exoplanet Orbit Database and the Exoplanet Data Explorer at exoplanets.org, the Extrasolar Planets Encyclopaedia at exoplanet.eu, and the SIMBAD and VizieR databases at simbad.u-strasbg.fr/simbad/ and http://vizier.u-strasbg.fr/viz-bin/VizieR.

\section{REFERENCES}

Albrecht, S., Winn, J. N., Johnson, J. A., et al. 2012, ApJ, 757, 18 Ammons, S. M., Robinson, S. E., Strader, J., et al. 2006, ApJ, 638,1004

Asplund, M., Grevesse, N., \& Sauval, A. J. 2005, in Astronomical Society of the Pacific Conference Series, Vol. 336, Cosmic Abundances as Records of Stellar Evolution and

Nucleosynthesis, ed. T. G. Barnes, III \& F. N. Bash, 25

Bakos, G., Noyes, R. W., Kovács, G., et al. 2004, PASP, 116, 266

Baranne, A., Queloz, D., Mayor, M., et al. 1996, A\&AS, 119, 373

Bieryla, A., Collins, K., Beatty, T. G., et al. 2015, AJ, 150, 12

Bouchy, F., Hébrard, G., Udry, S., et al. 2009, A\&A, 505, 853

Bourrier, V., Lecavelier des Etangs, A., Hébrard, G., et al. 2015, A\&A, 579, A55

Burke, C. J., McCullough, P. R., Valenti, J. A., et al. 2007, ApJ, 671,2115

-. 2008, ApJ, 686, 1331

Claret, A. 2000, A\&A, 363, 1081

-. 2004, A\&A, 428, 1001 
Collier Cameron, A., Bruce, V. A., Miller, G. R. M., Triaud, A. H. M. J., \& Queloz, D. 2010a, MNRAS, 403, 151

Collier Cameron, A., Bouchy, F., Hébrard, G., et al. 2007, MNRAS, 375, 951

Collier Cameron, A., Guenther, E., Smalley, B., et al. 2010b, MNRAS, 407, 507

Crouzet, N., McCullough, P. R., Deming, D., \& Madhusudhan, N. 2014, ApJ, 795, 166

Dawson, R. I., \& Chiang, E. 2014, Science, 346, 212

de Wit, J., \& Seager, S. 2013, Science, 342, 1473

Decressin, T., Mathis, S., Palacios, A., et al. 2009, A\&A, 495, 271

Deleuil, M., Deeg, H. J., Alonso, R., et al. 2008, A\&A, 491, 889

Deming, D., Wilkins, A., McCullough, P., et al. 2013, ApJ, 774, 95

Gandolfi, D., Hébrard, G., Alonso, R., et al. 2010, A\&A, 524, A55

Guillot, T., \& Havel, M. 2011, A\&A, 527, A20

Guillot, T., Santos, N. C., Pont, F., et al. 2006, A\&A, 453, L21

Hébrard, G., Evans, T. M., Alonso, R., et al. 2011, A\&A, 533, A130

Hog, E., Kuzmin, A., Bastian, U., et al. 1998, A\&A, 335, L65

Høg, E., Fabricius, C., Makarov, V. V., et al. 2000, A\&A, 355, L27

Holt, J. R. 1893, A\&A, 12, 646

Husnoo, N., Pont, F., Mazeh, T., et al. 2012, MNRAS, 422, 3151

Janes, K. A., \& Heasley, J. N. 1993, PASP, 105, 527

Johns-Krull, C. M., McCullough, P. R., Burke, C. J., et al. 2008, ApJ, 677, 657

Kovács, G., Zucker, S., \& Mazeh, T. 2002, A\&A, 391, 369

Kraft, R. P. 1967, ApJ, 150, 551

Lagarde, N., Decressin, T., Charbonnel, C., et al. 2012, A\&A, 543, A108

Lecavelier Des Etangs, A., Pont, F., Vidal-Madjar, A., \& Sing, D. 2008, A\&A, 481, L83

Leconte, J., Chabrier, G., Baraffe, I., \& Levrard, B. 2010, A\&A, $516, \mathrm{~A} 64$

Lehmann, H., Guenther, E., Sebastian, D., et al. 2015, A\&A, 578, L4

Lodders, K. 1999, ApJ, 519, 793

Mandel, K., \& Agol, E. 2002, ApJL, 580, L171

Mazeh, T., Nachmani, G., Sokol, G., Faigler, S., \& Zucker, S. 2012, A\&A, 541, A56

Mazeh, T., Zucker, S., \& Pont, F. 2005, MNRAS, 356, 955

McCullough, P. R., Crouzet, N., Deming, D., \& Madhusudhan, N. 2014, ApJ, 791, 55

McCullough, P. R., Stys, J. E., Valenti, J. A., et al. 2005, PASP, 117,783

-. 2006, ApJ, 648, 1228

McCullough, P. R., Burke, C. J., Valenti, J. A., et al. 2008, ArXiv e-prints
McLaughlin, D. B. 1924, ApJ, 60, 22

McQuillan, A., Mazeh, T., \& Aigrain, S. 2013, ApJL, 775, L11

Mislis, D., \& Hodgkin, S. 2012, MNRAS, 422, 1512

Moutou, C., Deleuil, M., Guillot, T., et al. 2013, Icarus, 226, 1625

Naoz, S., Farr, W. M., Lithwick, Y., Rasio, F. A., \& Teyssandier, J. 2011, Nature, 473, 187

Palacios, A., Charbonnel, C., Talon, S., \& Siess, L. 2006, A\&A, 453, 261

Palacios, A., Talon, S., Charbonnel, C., \& Forestini, M. 2003, A\&A, 399, 603

Parviainen, H., Deeg, H. J., \& Belmonte, J. A. 2013, A\&A, 550, A67

Pepe, F., Mayor, M., Galland, F., et al. 2002, A\&A, 388, 632

Pickles, A., \& Depagne, É. 2010, PASP, 122, 1437

Pollacco, D. L., Skillen, I., Collier Cameron, A., et al. 2006,

PASP, 118, 1407

Pont, F. 2009, MNRAS, 396, 1789

Pont, F., Sing, D. K., Gibson, N. P., et al. 2013, MNRAS, 432, 2917

Pont, F., Zucker, S., \& Queloz, D. 2006, MNRAS, 373, 231

Poppenhaeger, K., \& Wolk, S. J. 2014, A\&A, 565, L1

Rossiter, R. A. 1924, ApJ, 60, 15

Santerne, A., Moutou, C., Barros, S. C. C., et al. 2012, A\&A, $544, \mathrm{~L} 12$

Schlaufman, K. C. 2010, ApJ, 719, 602

Shporer, A., Jenkins, J. M., Rowe, J. F., et al. 2011, AJ, 142, 195

Shporer, A., O'Rourke, J. G., Knutson, H. A., et al. 2014, ApJ, 788,92

Siess, L., Dufour, E., \& Forestini, M. 2000, A\&A, 358, 593

Southworth, J. 2015, JKTLD: Limb darkening coefficients, Astrophysics Source Code Library

Southworth, J., Wheatley, P. J., \& Sams, G. 2007, MNRAS, 379, L11

Sozzetti, A., Torres, G., Charbonneau, D., et al. 2007, ApJ, 664, 1190

Tamuz, O., Mazeh, T., \& Zucker, S. 2005, MNRAS, 356, 1466

Teitler, S., \& Königl, A. 2014, ApJ, 786, 139

Torres, G., Winn, J. N., \& Holman, M. J. 2008, ApJ, 677, 1324

Valenti, J. A., \& Fischer, D. A. 2005, ApJS, 159, 141

Valenti, J. A., \& Piskunov, N. 1996, A\&AS, 118, 595

Valsecchi, F., \& Rasio, F. A. 2014, ApJ, 786, 102

Winn, J. N. 2010, Exoplanet Transits and Occultations, ed. S. Seager, $55-77$

Winn, J. N., Fabrycky, D., Albrecht, S., \& Johnson, J. A. 2010, ApJL, 718, L145

Winn, J. N., \& Fabrycky, D. C. 2015, ARA\&A, 53, 409

Winn, J. N., Holman, M. J., Torres, G., et al. 2008, ApJ, 683, 1076 